\title{
Factores asociados con dolor severo en pacientes con fascitis plantar. Un análisis de relaciones
}

\author{
Laura C. Gaitan, Ana B. Calvo, Valeria López, Juan Pablo Calvi, Victoria Álvarez, Gastón Slullitel \\ Unidad de Pie y Tobillo, Instituto de Ortopedia y Traumatología "Dr. Jaime Slullitel", Rosario, Santa Fe, Argentina
}

\begin{abstract}
RESUMEN
Objetivo: Describir los factores asociados con niveles de dolor más severo en una cohorte de pacientes con fascitis plantar. El objetivo secundario fue analizar cuáles de estos factores estaban asociados con niveles más altos de mejoría clínica luego del tratamiento conservador. Materiales y Métodos: Se evaluó a una cohorte prospectiva de pacientes con diagnóstico de fascitis plantar. Cada participante completó una escala ordinal visual de dolor (del 1 al 10) para dolor del primer paso y dolor al final del día y encuestas FFI-R (Foot Function Index-Revised). También se realizó una evaluación demográfica. La dorsiflexión de la articulación del tobillo, el rango de movilidad de la primera articulación metatarsofalángica, la rigidez del gastrocnemio y el ángulo poplíteo también se evaluaron de manera estándar. Resultados: Se incluyó a 214 pacientes. El 64\% eran hombres (118 pacientes), la media de la edad era de 49.67 años (DE 13.16) y el índice de masa corporal promedio, de 28,53 (DE 5,18). En el análisis multivariado, se observó que el riesgo de un puntaje $\geq 8$ en la escala de dolor aumentó cuando el paciente refirió estar de pie por más de $6 \mathrm{~h}$ (OR 1,$17 ; p=0,03 ;$ IC95\% 1,02-1,35). El riesgo de un puntaje >8 fue mayor cuando el grado de dorsiflexión del tobillo fue $<0^{\circ}(O R 1,20$; $p=0,03 ;$ IC95\% 1,02-1,41). Conclusión: Nuestros hallazgos apoyan indirectamente la hipótesis de que la dorsiflexión limitada del tobillo juega un papel como factor de riesgo asociado a un puntaje $\geq 8$ en la escala de dolor, en los casos de fascitis plantar.

Palabras clave: Fascitis plantar; talalgia; rigidez de gastrocnemios; fortalecimiento excéntrico; elongación.

Nivel de Evidencia: IV
\end{abstract}

Factors associated with severe pain in patients with plantar fasciitis: An association analysis

\begin{abstract}
Objective: The main purpose of our study was to describe the factors associated with more severe pain levels in a cohort of patients with plantar fasciitis (PF). The secondary purpose of this study was to determine which of these factors were associated with higher levels of clinical improvement after conservative therapy. Materials and Methods: We conducted a prospective study in a cohort of patients with PF. Each participant completed an ordinal pain scale (1-10) for first-step pain and end-of-day pain, and Foot Function Index-Revised (FFI-R) surveys at enrollment. Also, patient demographics were evaluated. The ankle joint dorsiflexion, the range of motion (ROM) for the first metatarsophalangeal joint (MTPJ), the gastrocnemius tightness, and the popliteal angle were evaluated through standard tests. Results: Our study included 214 participants, of which $64 \%$ (118 patients) were males, the average age was 49.67 years (SD 13.16) and the average BMI was 28.53 (SD 5.18). The multivariate analysis showed that the risk of having a Visual Analog Scale (VAS) score $\geq 8$ increased when the patient reported standing for more than 6 hours $(\mathrm{OR}=1.17$; $\mathrm{P}=0.03$; Cl95\%: 1.02-1.359). The risk of a $>8$-VAS score was higher when the level of ankle dorsiflexion was $<0(O R=1.20 ; P=0.03$; CI95\%: 1.02-1.41). Conclusion: Our findings indirectly support the hypothesis that limited ankle dorsiflexion ROM plays a role as a risk factor associated with VAS scores $\geq 8$ in PF patients.
\end{abstract}

Key words: Plantar fasciitis; heel pain; gastrocnemius tightness; eccentric strengthening; stretching.

Level of Evidence: IV

Recibido el 16-5-2019. Aceptado luego de la evaluación el 19-8-2019 • Dra. LAURA C. GAITAN • Icgaitan3@gmail.com (ID

Cómo citar este artículo: Gaitan LC, Calvo AB, López V, Calvi JP, Álvarez V, Slullitel G. Factores asociados con dolor severo en pacientes con fascitis plantar. Un análisis de relaciones. Rev Asoc Argent Ortop Traumatol 2020;85(1):23-30. https://doi.org/10.15417/issn.1852-7434.2020.85.1.991 


\section{INTRODUCCIÓN}

La fascitis plantar (FP) es la causa más común de dolor plantar en el retropié, ${ }^{1,2}$ y tiene un impacto negativo significativo en la percepción de la salud general. ${ }^{3}$ Los síntomas típicos incluyen dolor en la zona plantar medial del talón, particularmente durante los primeros pasos al comienzo del día, que mejora progresivamente con la deambulación. Más aún, el dolor, en ocasiones, empeora con la estancia prolongada de pie o con el reposo. ${ }^{4}$

Desde la teoría, se han relacionado múltiples factores con este cuadro, como limitación de la movilidad metatarsofalángica, limitación del rango de movilidad del tobillo, acompañados de influencias ambientales y circunstanciales. No obstante, la influencia de estos factores en el desarrollo de la FP posee evidencia empírica aún pobre en la bibliografía. Los autores de este artículo no conocen que haya evidencia que analice la severidad del dolor en esta patología, en particular.

Una revisión clínica de FP llevada a cabo por $\operatorname{Orchad}^{5}$ describe cinco categorías de tratamiento recomendadas, evaluadas o revisadas: biomecánico (que incluye ortesis, modificaciones del calzado y tapping), técnicas de elongación (que incluyen férulas nocturnas), ondas de choque extracorpóreas, inyecciones de corticoides e intervenciones quirúrgicas. A pesar de esto, no existe un algoritmo establecido y la evidencia de buena calidad es escasa.

El propósito principal de nuestro estudio fue describir los factores asociados con niveles de dolor más severo, en una cohorte de pacientes con FP. El objetivo secundario fue analizar cuáles de estos factores estaban asociados con niveles más altos de mejoría clínica luego del tratamiento conservador.

\section{MATERIALES Y MÉTODOS}

\section{Participantes}

Entre diciembre de 2017 y octubre de 2018, se evaluó a una cohorte prospectiva de pacientes con diagnóstico de FP y sin antecedente de traumatismo localizado en esa región, quienes consintieron voluntariamente en participar en el estudio.

Se incluyó a pacientes con diagnóstico de FP según los siguientes criterios: dolor en la región plantar medial del talón con irradiación al borde medial del arco del pie o sin irradiación, que se puede incrementar con los primeros pasos del día o luego de la estancia de pie por algunas horas. Todos los pacientes con enfermedades neuromusculares o antecedente reciente de trauma o cirugía en el miembro inferior fueron excluidos.

\section{Mediciones}

Cada participante completó una escala ordinal visual de dolor (de 1 a 10) durante el primer paso del día y al final del día, y el cuestionario FFI-R (Foot Function Index-Revised) en el momento de la incorporación. Este índice validado mide el impacto de la patología del pie en la función, en términos de dolor, incapacidad y restricción de actividades. El paciente debía puntuar cada pregunta en una escala de 0 (sin dolor ni dificultad) a 10 (peor dolor inimaginable o tan dificultoso que requiere de asistencia), de modo que describa, de manera más precisa, el dolor en el pie durante la semana previa. ${ }^{6,7}$

Asimismo, se registró información sobre otras variables potenciales que incluían edad, sexo, duración de los síntomas, talla, peso, lateralidad, tipo de pie y cuadros médicos previos.

La dorsiflexión del tobillo fue medida sin carga con la rodilla extendida. El paciente fue colocado en posición supina y con la articulación subastragalina en ligera inversión. El goniómetro fue alineado de manera que un brazo biseccione la diáfisis del peroné en el lado lateral de la pierna y el otro brazo esté alineado con el borde lateral del pie. El tobillo fue colocado en dorsiflexión manualmente hasta la primera resistencia. La dorsiflexión entre $-15^{\circ}$ y $0^{\circ}$ fue considerada como no limitada y entre $1^{\circ}$ y $25^{\circ}$, limitada.

El rango de movilidad de la primera articulación metatarsofalángica se determinó clínicamente con el pie en carga con presión plantar hasta el antepié. Las mediciones se tomaron con un goniómetro, con la línea medioaxial de la falange proximal y la superficie plantar del pie como puntos de referencia. ${ }^{8}$

La rigidez del gastrocnemio fue evaluada mediante la prueba de Silfverskiöld: equino del tobillo cuando se extiende la rodilla, pero que desaparece cuando la rodilla se flexiona durante la aplicación de una carga con una fuerza moderada debajo del antepié; y se normaliza con la rodilla en flexión. ${ }^{9}$

El ángulo poplíteo busca la elasticidad de los músculos isquiotibiales. Con el paciente acostado en posición supina sobre la mesa de examen, con la cadera y la rodilla flexionadas a $90^{\circ}$, la rodilla se extendió gradualmente hasta buscar la sensación de malestar debido a la resistencia firme, en la cara posterior del muslo. En ese momento, se midió el ángulo formado por la bisección lateral del muslo y la pierna. El centro del goniómetro fue colocado en la cara lateral de la rodilla, coincidiendo con el eje de rotación. Un brazo se colocó paralelo a la bisección lateral 
del muslo y el otro se dirigió hacia el maléolo lateral. El ángulo de flexión residual de la rodilla se midió como los grados que faltan desde la extensión completa $\left(180^{\circ}\right)$. Se consideró como rango normal de $0^{\circ}$ a $15^{\circ}$, mientras que los valores de $16^{\circ}$ a $34^{\circ}$ corresponden a contractura moderada de los isquiotibiales, y los valores $\geq 35^{\circ}$, a contractura severa. ${ }^{10}$

Todos los pacientes fueron examinados por un solo cirujano especializado en pie y tobillo, y completaron todos los cuestionarios sobre el pie estudiado en su visita de seguimiento de las semanas 6-14.

La satisfacción del paciente con el tratamiento aplicado se evaluó preguntándole si lo recomendaría a un familiar o amigo.

\section{Protocolo de tratamiento}

Todos siguieron un protocolo de tratamiento que consistió en la liberación miofascial del tríceps sural y fascia plantar, automovilización del tobillo en dorsiflexión y flexibilidad dinámica en la cadena posterior, desplazamiento del centro de gravedad bípode y unipodal, fortalecimiento excéntrico del gastrocnemio bilateral y un masaje profundo en la fascia plantar en busca de dolor supramáximo. Se los instruyó para que, en el hogar, realicen un tratamiento criogénico, tres veces por día, haciendo rodar una botella congelada debajo de la planta del pie (ejerciendo presión) y elongación suave del gastrocnemio y la fascia plantar. Se les indicó que hicieran todas las actividades de la vida diaria que no aumentaran los síntomas y que evitaran las actividades agravantes. Este protocolo se aplicó durante un período de seis semanas.

\section{Análisis estadístico}

Las variables categóricas se describen como valor absoluto (n) y valor relativo (\%), y las variables continuas, como media y desviación estándar (DE) o mediana y rango intercuartílico según la distribución. La distribución se evaluó mediante gráficos de dispersión y la prueba de Shapiro-Wilk. Clasificamos el grado de dorsiflexión del tobillo en cuatro categorías ( $>10$; de $5 \mathrm{a} \leq 10$; de 0 a $\leq 5$ y $\leq 0$ ) teniendo en cuenta el artículo publicado por Riddle y cols. para una mejor interpretación. ${ }^{11}$

Para evaluar los factores asociados con una mayor intensidad del dolor, la variable del puntaje de la escala analógica visual (EAV) se dicotomizó en $\geq 8 \mathrm{y}<8$. A fin de evaluar a los pacientes que respondieron al tratamiento, los clasificamos según el punto de corte de $\Delta \mathrm{EAV} \geq 4$. Teniendo en cuenta que había participantes con fascitis bilateral y que los casos se anidaron por paciente, el análisis bivariado y multivariado se llevó a cabo mediante un modelo de regresión de la ecuación de estimación generalizada, con una matriz de correlación intercambiable, considerando a cada paciente como grupo. En el análisis multivariado, se verificaron posibles interacciones entre variables. Los modelos finales fueron probados por criterios QIC. El nivel de significación se consideró $\mathrm{p}<0,05$. Los análisis estadísticos se realizaron con el programa Stata 13.0.

Para estudiar el posible sesgo debido a la pérdida de seguimiento, se compararon las características iniciales de los pacientes que cumplieron el seguimiento frente a aquellos perdidos durante el seguimiento.

\section{RESULTADOS}

Se incorporaron 182 pacientes con FP, 32 (17,52\%) tenían fascitis bilateral, lo que dio un total de 214 casos. El 64\% (118 pacientes) eran hombres, con una edad promedio de 49.67 años (DE 13.16) y un índice de masa corporal promedio de 28,53 (DE 5,18). Las características basales se describen en la Tabla 1. Las características anatómicas, funcionales y relacionadas con el dolor se muestran en la Tabla 2.

\section{Factores asociados a un dolor más intenso}

De acuerdo con el punto de corte de la EAV $\geq 8$ al inicio del estudio, 118 casos tenían un puntaje $\geq 8$ y 96 pacientes, $<8$ (Tabla 3). En el análisis multivariado, observamos que el riesgo de un puntaje $\geq 8$ aumentó cuando el paciente reportó estar de pie por más de 6 h (razón de probabilidades [odds ratio, OR] 1,17; p = 0,03; IC95\% $1,02-1,35)$. Cuando el grado de dorsiflexión del tobillo era $<0$, el riesgo de un puntaje $>8$ fue mayor (OR 1,$20 ; p$ $=0,03$; IC95\% 1,02-1,41). Una consulta previa por la FP se asocia con un puntaje basal $<8(\mathrm{OR} 0,79 ; \mathrm{p}=0,001$; IC95\% 0,69-0,91).

\section{Seguimiento}

En el seguimiento final, se perdieron 28 participantes, lo que resultó en 154 pacientes y 186 casos. La mediana de seguimiento fue de 8.5 semanas (rango intercuartílico: 6-14). 
Tabla 1. Características basales de los pacientes incluidos $(\mathrm{n}=182)$

\begin{tabular}{|l|c|}
\hline Características & \\
\hline Sexo (masculino) & $118(64,84 \%)$ \\
\hline Edad $^{+}$ & $49.67(13.16)$ \\
\hline Índice de masa corporal $^{+}$ & $28,52(5,18)$ \\
\hline Hábito tabáquico & $21(11,54 \%)$ \\
\hline Comorbilidades & $66(36,26 \%)$ \\
\hline Bipedestación por más de $6 \mathrm{~h}$ & $111(60,99 \%)$ \\
\hline Consultas previas & $77(42,31 \%)$ \\
\hline Tratamientos previos & \\
\hline Sin tratamiento & $126(69,23 \%)$ \\
\hline Fisioterapia con elongación & $19(10,44 \%)$ \\
\hline Fisioterapia sin elongación & $27(14,84 \%)$ \\
\hline Infiltración con corticoides & $10(5,49 \%)$ \\
\hline Seguimiento & $154(84,62 \%)$ \\
\hline Fascitis bilateral & $32(17,52 \%)$ \\
\hline
\end{tabular}

+Media (DE).

Tabla 2. Características basales de los casos de fascitis plantar $(\mathrm{n}=214)$

\begin{tabular}{|c|c|}
\hline \multicolumn{2}{|l|}{ Características } \\
\hline Pie izquierdo & $107(50 \%)$ \\
\hline $\begin{array}{l}\text { Tipo de pie } \\
\text { Neutro } \\
\text { Cavo } \\
\text { Pie plano }\end{array}$ & $\begin{array}{c}103(48,13 \%) \\
67(31,31 \%) \\
44(20,56 \%)\end{array}$ \\
\hline Dolor atípico & $21(9,81 \%)$ \\
\hline $\begin{array}{l}\text { Localización del dolor } \\
\text { Insercional medial } \\
\text { Insercional central } \\
\text { No insercional }\end{array}$ & $\begin{array}{c}107(50 \%) \\
87(40,65 \%) \\
20(9,35 \%)\end{array}$ \\
\hline Irradiación del dolor & $12(5,61 \%)$ \\
\hline Dorsiflexión limitada de la primera articulación metatarsofalángica & $64(29,91 \%)$ \\
\hline Prueba de Silfverskiöld positiva & $24(11,21 \%)$ \\
\hline Dorsiflexión de tobillo $\left(^{\circ}\right)$ & $10(0-15)$ \\
\hline $\begin{array}{l}\text { Dorsiflexión de tobillo }\left(^{\circ}\right) \\
>10 \\
5-\geq 10 \\
0-\geq 5 \\
\leq 0\end{array}$ & $\begin{array}{l}86(40,19 \%) \\
40(18,69 \%) \\
25(11,68 \%) \\
63(29,44 \%)\end{array}$ \\
\hline Ángulo poplíteo ${ }^{+}$ & $32,03(16,55)$ \\
\hline $\mathrm{EAV}^{*}$ & $8(6-9)$ \\
\hline $\mathrm{FFI}(\%)^{+}$ & $45,89(10,88)$ \\
\hline
\end{tabular}

${ }^{+}$Media (DE), *mediana (percentilo 25-75), EAV = escala analógica visual, FFI = Functional Foot Index. 
Tabla 3. Factores de riesgo asociados a EAV $\geq 8$ en casos de fascitis plantar

\begin{tabular}{|c|c|c|c|c|}
\hline & $\begin{array}{c}\text { Cantidad de } \\
\text { casos con EAV } \\
\geq 8 \\
(n=118)\end{array}$ & $\begin{array}{c}\text { Cantidad de } \\
\text { controles con } \\
\text { EAV }<8 \\
(\mathbf{n}=\mathbf{9 6})\end{array}$ & OR (valor p; IC95\%) & OR ajustado \\
\hline Edad $^{+}$ & $50.34(13.3)$ & $48.85(13.01)$ & $1(0,41 ; 0,99-1,007)$ & \\
\hline Sexo masculino & $36(30,51 \%)$ & $36(37,5 \%)$ & $0,90(0,176 ; 0,78-1,04)$ & \\
\hline $\begin{array}{l}\text { Índice de masa corporal } \\
\leq 25 \\
>25-\leq 30 \\
>30\end{array}$ & $\begin{array}{l}29(24,58 \%) \\
50(42,37 \%) \\
39(33,05 \%)\end{array}$ & $\begin{array}{l}22(22,92 \%) \\
41(42,71 \%) \\
33(34,88 \%)\end{array}$ & $\begin{array}{c}1 \\
0,96(0,66 ; 0,80-1,15) \\
0,98(0,80 ; 0,80-1,18)\end{array}$ & \\
\hline Comorbilidades & $41(34,75 \%)$ & $35(36,46 \%)$ & $0,97(0,71 ; 0,84-1,12)$ & \\
\hline Consultas previas & $39(33,95 \%)$ & $52(54,17 \%)$ & $0,78(0,001 ; 0,68-0,9)$ & $0,79(0,001 ; 0,69-0,91)$ \\
\hline $\begin{array}{l}\text { Tipo de pie } \\
\text { Neutro } \\
\text { Cavo } \\
\text { Pie plano }\end{array}$ & $\begin{array}{l}56(47,46 \%) \\
33(27,97 \%) \\
29(24,58 \%)\end{array}$ & $\begin{array}{l}47(48,96 \%) \\
34(35,42 \%) \\
15(15,63 \%)\end{array}$ & $\begin{array}{c}1 \\
0,94(0,40 ; 0,80-1,09) \\
1,15(0,12 ; 0,96-1,37)\end{array}$ & \\
\hline Bipedestación por más de $6 \mathrm{~h}$ & $79(66,95 \%)$ & $53(55,21 \%)$ & $1,19(0,019 ; 1,03-1,37)$ & $1,17(0,03 ; 1,02-1,35)$ \\
\hline \multicolumn{5}{|l|}{ Fascitis bilateral } \\
\hline Dolor atípico & $11(9,32 \%)$ & $10(10,42 \%)$ & $0,96(0,75-1,23)$ & \\
\hline $\begin{array}{l}\text { Localización del dolor } \\
\text { Insercional medial } \\
\text { Insercional central } \\
\text { No insercional }\end{array}$ & $\begin{array}{c}52(44,07 \%) \\
55(46,61 \%) \\
11(9,32 \%)\end{array}$ & $\begin{array}{c}55(57,29 \%) \\
32(33,33 \%) \\
9(9,38 \%)\end{array}$ & $\begin{array}{c}1 \\
1,10(0,15 ; 0,96-1,28) \\
0,97(0,78 ; 0,76-1,23)\end{array}$ & \\
\hline Irradiación del dolor & $8(6,78 \%)$ & $4,17(4 \%)$ & $1,1(0,46 ; 0,84-1,45)$ & \\
\hline $\begin{array}{l}\text { Dorsiflexión limitada } \\
\text { de la primera articulación } \\
\text { metatarsofalángica }\end{array}$ & $38(32,20 \%)$ & $26(27,08 \%)$ & $1,06(0,43 ; 0,91-1,24)$ & \\
\hline $\begin{array}{l}\text { Prueba de Silfverskiöld } \\
\text { positiva }\end{array}$ & $10(8,47 \%)$ & $14(14,58 \%)$ & $0,85(0,127 ; 0,69-1,05)$ & \\
\hline $\begin{array}{l}\text { Dorsiflexión de tobillo }\left({ }^{\circ}\right) \\
10 \\
5-\leq 10 \\
0-\leq 5 \\
\leq 0\end{array}$ & $\begin{array}{l}38(32,20 \%) \\
24(20,34 \%) \\
14(11,86 \%) \\
42(35,59 \%)\end{array}$ & $\begin{array}{c}48(50 \%) \\
16(16,67 \%) \\
11(11,46 \%) \\
21(21,88 \%)\end{array}$ & $\begin{array}{c}1 \\
1,15(0,12 ; 0,96-1,39) \\
1,11(0,34 ; 0,89-1,38) \\
1,25(0,007 ; 1,06-1,48)\end{array}$ & $\begin{array}{l}1,15(0,07 ; 0,99-1,35) \\
1,09(0,40 ; 0,89-1,33) \\
1,20(0,03 ; 1,02-1,41)\end{array}$ \\
\hline Ángulo poplíteo ${ }^{+}$ & $30,35(1,42)$ & $33,4(1,69)$ & $1,00(0,18 ; 0,99-1,006)$ & \\
\hline
\end{tabular}

${ }^{+}$Media (DE), EAV = escala analógica visual.

OR ajustado por bipedestación por más de $6 \mathrm{~h}$, dorsiflexión de tobillo $\left({ }^{\circ}\right)$, consultas previas.

Los cambios en la severidad del dolor se expresaron como $\triangle \mathrm{EAV}$. Establecimos un punto de corte de $\Delta \mathrm{EAV} \geq 4$, por lo que 118 casos fueron clasificados como $\triangle \mathrm{EAV} \geq 4$ y 68 controles, $\triangle \mathrm{EAV}<4$. Cuando evaluamos los factores relacionados con un cambio en el puntaje de la EAV $\geq 4$, no hallamos diferencias significativas en ambos grupos teniendo en cuenta el sexo, la edad, el índice de masa corporal o las características clínicas. No obstante, observamos que las posibilidades de mejorar más de 4 puntos en la EAV se reducen un $20 \%$ para aquellos con el ángulo de dorsiflexión del tobillo $<0^{\circ}$ (Tabla 4). 
Tabla 4. Factores asociados a una $\triangle \mathrm{EAV} \geq 4$ después de un tratamiento estandarizado

\begin{tabular}{|c|c|c|c|}
\hline & $\begin{array}{l}\text { Cantidad de casos } \\
\text { con } \triangle E A V \geq 4 \\
(n=118)\end{array}$ & $\begin{array}{l}\text { Cantidad de casos } \\
\text { con } \triangle E A V<4 \\
(n=68)\end{array}$ & OR (valor p; IC95\%) \\
\hline Edad $^{+}$ & $50.55(12.6)$ & $48.39(13.83$ & $1(0,28 ; 0,99-1,003)$ \\
\hline Sexo masculino & $39(33,05 \%)$ & $20(29,41 \%)$ & $0,97(0,708 ; 0,83-1,13)$ \\
\hline $\begin{array}{l}\text { Índice de masa corporal } \\
\leq 25 \\
>25-\leq 30 \\
>30\end{array}$ & $\begin{array}{l}29(24,58 \%) \\
50(42,37 \%) \\
39(33,05 \%)\end{array}$ & $\begin{array}{c}17(25 \%) \\
28(41,18 \%) \\
23(33,82 \%)\end{array}$ & $\begin{array}{c}1 \\
1,01(0,90 ; 0,84-1,21) \\
1(0,99 ; 0,83-1,21)\end{array}$ \\
\hline Comorbilidades & $42(35,59 \%)$ & $28(41,18 \%)$ & $1,05(0,48 ; 0,91-1,22)$ \\
\hline Consultas previas & $44(37,29 \%)$ & $31(45,59 \%)$ & $1,08(0,26 ; 0,94-1,25)$ \\
\hline Tratamiento previo & $32(27,12 \%)$ & $19(27,94 \%)$ & $1,02(0,71 ; 0,88-1,20)$ \\
\hline $\begin{array}{l}\text { Tipo de pie } \\
\text { Neutro } \\
\text { Cavo } \\
\text { Pie plano }\end{array}$ & $\begin{array}{c}59(50 \%) \\
34(28,81 \%) \\
25(21,19 \%)\end{array}$ & $\begin{array}{l}33(48,53 \%) \\
22(32,35 \%) \\
13(19,12 \%)\end{array}$ & $\begin{array}{c}1 \\
1,03(0,71 ; 0,87-1,22) \\
0,99(0,90 ; 0,83-1,18)\end{array}$ \\
\hline Bipedestación por más de $6 \mathrm{~h}$ & $73(61,86 \%)$ & $37(54,41 \%)$ & $0,94(0,496 ; 0,82-1,10)$ \\
\hline Dolor atípico & $8(6,78 \%)$ & $8(11,76 \%)$ & $1,1(0,83-1,46)$ \\
\hline $\begin{array}{l}\text { Localización del dolor } \\
\text { Insercional medial } \\
\text { Insercional central } \\
\text { No insercional }\end{array}$ & $\begin{array}{c}60(50,85 \%) \\
49(41,53 \%) \\
9(7,63 \%)\end{array}$ & $\begin{array}{c}31(45,59 \%) \\
28(41,18 \%) \\
9(13,24 \%)\end{array}$ & $\begin{array}{c}1 \\
1,06(0,49 ; 0,91-1,23) \\
1,13(0,36 ; 0,87-1,47)\end{array}$ \\
\hline Irradiación del dolor & $5(4,24 \%)$ & $6(8,82 \%)$ & $1,23(0,181 ; 0,91-1,68)$ \\
\hline $\begin{array}{l}\text { Dorsiflexión limitada de la primera } \\
\text { articulación metatarsofalángica }\end{array}$ & $38(32,20 \%)$ & $19(27,94 \%)$ & $0,98(0,77 ; 0,84-1,14)$ \\
\hline Prueba de Silfverskiöld positiva & $13(11,02 \%)$ & $11(16,18 \%)$ & $1,12(0,26 ; 0,92-1,36)$ \\
\hline $\begin{array}{l}\text { Dorsiflexión de tobillo } \\
10 \\
5-\leq 10 \\
0-\leq 5 \\
\leq 0\end{array}$ & $\begin{array}{l}46(38,98 \%) \\
21(17,80 \%) \\
13(11,02 \%) \\
38(32,20 \%)\end{array}$ & $\begin{array}{c}36(52,94 \%) \\
14(20,59 \%) \\
7(10,29 \%) \\
11(16,18 \%)\end{array}$ & $\begin{array}{c}1 \\
0,97(0,77 ; 0,81-1,17) \\
0,93(0,52 ; 0,74-1,16) \\
0,81(0,02 ; 0,68-0,96)\end{array}$ \\
\hline Ángulo poplíteo ${ }^{+}$ & $30,35(1,42)$ & $33,4(1,69)$ & $1,00(0,76 ; 0,996-1,004)$ \\
\hline
\end{tabular}

${ }^{+}$Mediana (DE), EAV = escala analógica visual.

La satisfacción de los pacientes fue excelente en 66 casos (35,48\%), buena en 83 (44,62\%), regular en 30 $(16,13 \%)$ y mala en $7(3,76 \%)$. Ninguna de las variables analizadas se relacionó significativamente con el grado de satisfacción.

\section{DISCUSIÓN}

La FP es un cuadro habitual que representa aproximadamente entre el $11 \%$ y el $15 \%$ de todas las molestias del pie que requieren evaluación médica. ${ }^{1,3}$

En la bibliografía, se han señalado muchos factores para el dolor crónico del talón y comúnmente se caracterizan como intrínsecos o extrínsecos. Los factores intrínsecos son aquellos propios de un individuo que lo predisponen a lesionarse. ${ }^{12}$ Los sugeridos en la bibliografía incluyen rango de movilidad limitado de la primera articulación metatarsofalángica, dorsiflexión del tobillo limitada, discrepancia en la longitud de la pierna, reducción del grosor 
de la almohadilla plantar, aumento del grosor de la fascia plantar, excesiva pronación del pie, reducción de la fuerza de la pantorrilla, edad avanzada y aumento del índice de masa corporal. ${ }^{13-15}$ Las influencias ambientales y coyunturales que actúan sobre un individuo se conocen como factores extrínsecos e incluyen la bipedestación prolongada, el uso de calzado inadecuado, lesión previa y también influyen la superficie de carrera, la velocidad, la frecuencia y la distancia semanal en los deportistas. ${ }^{1,13,14}$ La evidencia empírica para la mayoría de estos factores es limitada o nula, ${ }^{1}$ lo que significa que el papel (si lo hay) de cada uno de estos factores en el desarrollo del dolor crónico del talón no se conoce bien. ${ }^{16}$ Según nuestro conocimiento, no hay evidencia publicada que analice la relación entre la gravedad de los síntomas y el grado de mejoría clínica.

Los hallazgos de este estudio apoyan indirectamente la hipótesis de que la dorsiflexión limitada del tobillo desempeña un papel como factor de riesgo asociado a un puntaje $\geq 8$ en la EAV, en los casos de FP. La limitación en la dorsiflexión del tobillo puede deberse a una contractura del complejo gastrocnemio-sóleo o a una contractura aislada del músculo gastrocnemio. Nuestros hallazgos coinciden con los de Riddle y cols., quienes, en un estudio pareado de casos y controles, informan que la dorsiflexión limitada del tobillo en el lado afectado incrementó significativamente el riesgo de FP después de ajustar para las otras variables en el modelo evaluado. Además, los sujetos que informaron que permanecían de pie durante la mayor parte de la jornada laboral también tuvieron un riesgo significativamente mayor de FP (OR 3,6; $p<0,05$; IC95\% 1,3-10,1). ${ }^{11,17}$

Patel y cols. estudiaron la proporción de pacientes con FP y una contractura aislada asociada del gastrocnemio. Este grupo halló que el 57\% (145 de 254) de los pacientes con FP tenía una contractura aislada del gastrocnemio; el 26\% (66 de 254), una contractura del complejo gastrocnemio-sóleo y el 17\% (43 de 254) no tenía limitación de la dorsiflexión del tobillo. ${ }^{1}$

En nuestra población, los pacientes con severo equino de la articulación del tobillo tenían más probabilidades de responder favorablemente a un programa de tratamiento que se centraba en el cuidado conservador y que incluyera la elongación del tendón de Aquiles. Nuestros hallazgos coinciden con los de un estudio previo que analiza el hecho de que un mayor equino del tobillo al comienzo de la terapia física se traduce en un mejor resultado después del tratamiento conservador, ${ }^{15}$ probablemente resaltando la importancia de un programa de elongación del tendón de Aquiles como parte integral del manejo conservador de la FP..$^{16-18}$

Fuera de la limitación de la dorsiflexión del tobillo, no hallamos ninguna otra medida biomecánica asociada con la respuesta al tratamiento. Aunque se ha atribuido la rigidez de los isquiotibiales en el desarrollo de la $\mathrm{FP},{ }^{12,19,20}$ la flexión de la cadera no se correlacionó con la probabilidad de lograr una respuesta favorable en nuestros pacientes. Sin embargo, este hallazgo no fue del todo inesperado, dado que, en nuestro protocolo de tratamiento, no existe ningún gesto específico destinado a abordar una posible contractura de los músculos posteriores de la extremidad proximal.

La bipedestación prolongada en el trabajo también se asoció con la aparición de FP en nuestra población.

De alguna manera, sorprendió el hallazgo de que el hallux limitus o el hallux limitus funcional no se asociaron con la aparición de FP ni con la respuesta al tratamiento; a pesar de que, en un estudio de casos y controles, Aranda y Munuera informaron significativamente más dorsiflexión de la articulación metatarsofalángica del primer metatarsiano en un grupo de control comparado con aquellos con FP $\left(71^{\circ}\right.$ vs. $\left.49^{\circ}\right) .^{12}$

Finalmente, encontramos que los pacientes con dolor intenso en el talón $(>8 / 10)$ tenían más probabilidades de responder favorablemente a las medidas de tratamiento conservador. Esto significa que los pacientes que acudieron para el tratamiento de síntomas leves o moderados respondieron menos a las terapias conservadoras para el dolor en el talón. En una condición, en gran medida autolimitada, con un alto efecto de tratamiento con placebo, ${ }^{2}$ no es sorprendente ver que los pacientes con el máximo dolor pueden tener más probabilidades de experimentar alivio con el inicio de un protocolo de elongación.

Las limitaciones de este estudio incluyen la falta de una población de control, como es la falta de un patrón de referencia ampliamente aceptado para la evaluación del rango de movilidad del tobillo. Además, aunque perdimos pacientes durante el seguimiento, aquellos que siguieron y los que se perdieron durante el seguimiento tenían características basales similares, por lo que consideramos que la pérdida durante el seguimiento fue aleatoria y no creó ningún sesgo.

El diseño de este estudio no permite establecer una relación de causa y efecto, ya que no proporciona datos sobre las relaciones temporales entre la presencia de FP y las variables medidas, mediante la comparación de cohortes, por lo que los factores evaluados como asociados con la FP pueden no ser los únicos involucrados.

Se deberán llevar a cabo estudios clínicos que se centren en el tiempo requerido para responder al tratamiento conservador y estimar el alivio de los síntomas en cada presentación diferente de la FP, en un intento por predecir la evolución de esta patología. 
ORCID de A. B. Calvo: http://orcid.org/0000-0001-5784-6144 ORCID de V. López: http://orcid.org/0000-0001-6345-5991 ORCID de J. P. Calvi: http://orcid.org/0000-0002-2738-0125
ORCID de V. Álvarez: http://orcid.org/0000-0002-8385-0128 ORCID de G. Slullitel: http://orcid.org/0000-0002-4842-7447

\section{BIBLIOGRAFÍA}

1. Crawford F, Thomson C. Interventions for treating plantar heel pain. Cochrane Database Syst Rev 2003;(3):CD000416. https://doi.org/10.1002/14651858.CD000416

2. Tisdel CL, Donley BG, Sferra JJ. Diagnosing and treating plantar fasciitis: a 511 conservative approach to plantar heel pain. Cleve Clin J Med 1999;66(4):231-5. https://doi.org/10.3949/ccjm.66.4.231

3. Irving DB, Cook JL, Young MA, Menz HB. Impact of chronic plantar heel pain 524 on health-related quality of life. J Am Podiatr Med Assoc 2008;98(4):283-9. https://doi.org/10.7547/0980283

4. Arslan A, Koca TT, Utkan A, Sevimli R, Akel I. Treatment of chronic plantar heel pain 310 with radiofrequency neural ablation of the first branch of the lateral plantar nerve and 311 medial calcaneal nerve branches. J Foot Ankle Surg 2016;55:767-71. https://doi.org/10.1053/j.jfas.2016.03.009

5. Orchard J. Plantar fasciitis-Clinical review. BMJ 2012;345:e6603. https://doi.org/10.1136/bmj.e6603

6. Bennett PJ, Patterson C, Wearing S, Baglioni T. Development and validation of a questionnaire designed to measure foot-health status. J Am Podiatr Med Assoc 1998;88(9):419-28. https://doi.org/10.7547/87507315-88-9-419

7. Reips UD, Funke F. Interval-level measurement with visual analogue scales in Internet-based research: VAS Generator. Behavior Research Methods 2008;40(3):699-704. https://doi.org/10.3758/BRM.40.3.699

8. Coughlin MJ, Shurnas PS. Hallux rigidus: demographics, etiology, and radiographic assessment. Foot Ankle Int 2003;24:731-43. https://doi.org/10.1177/107110070302401002

9. Silfverskiöld N. Reduction of the uncrossed two-joints muscles of the leg to one-joint muscles in spastic conditions. Acta Chir Scand 1924;56:315-28.

10. Quintana E, Albuquerque F. Evidencia científica de los métodos de evaluación de la elasticidad de la musculatura isquiotibial. Osteopat Cient 2008;3:115-24. https://doi.org/10.1016/S1886-9297(08)75760-6

11. Riddle DL, Pulisic M, Pidcoe P, Johnson RE. Risk factors for plantar fasciitis: a matched case-control study. J Bone Joint Surg Am 2003;85(5):872-7. https://doi.org/10.2106/00004623-200305000-00015

12. Aranda Y, Munuera PV. Plantar fasciitis and its relationship with hallux limitus. J Am Podiatr Med Assoc 2014; 104(3):263-8. https://doi.org/10.7547/0003-0538-104.3.263

13. Amis J, Jennings L, Graham D, Graham CE. Painful heel syndrome: radiographic and treatment assessment. Foot Ankle 1988;9(2):91-5. https://doi.org/10.1177/107110078800900206

14. Taunton JE, Ryan MB, Clement DB, McKenzie DC, Lloyd-Smith DR. Plantar fasciitis: a retrospective analysis of 267 cases. Phys Ther Sport 2002;3:57-65. https://doi.org/10.1054/ptsp.2001.0082

15. Rome K, Howe T, Haslock I. Risk factors associated with the development of plantar heel pain in athletes. Foot 2001;11:119-25. https://doi.org/10.1054/foot.2001.0698

16. Cole C, Seto C, Gazewood J. Plantar fasciitis: evidence-based review of diagnosis and therapy. Am Fam Physician 2005;72(11):2237-42. PMID: 16342847

17. Landorf KB, Menz HB. Plantar heel pain and fasciitis. BMJ Clin Evid 2008. pii: 1111. PMID: 19450330

18. Sweeting D, Parish B, Hooper L, Chester R. The effectiveness of manual stretching in the treatment of plantar heel pain: a systematic review. J Foot Ankle Res 2011;4:19. https://doi.org/10.1186/1757-1146-4-19

19. Bolívar YA, Munuera PV, Padillo JP. Relationship between tightness of the posterior muscles of the lower limb and plantar fasciitis. Foot Ankle Int 2013;34(1):42-8. https://doi.org/10.1177/1071100712459173

20. Budiman-Mak E, Conrad K, Stuck R, Matters M. Theoretical model and Rasch analysis to develop a revised Foot Function Index. Foot Ankle Int 2006;27(7):519-27. https://doi.org/10.1177/107110070602700707 